\title{
Functional evaluation of patients with mastectomy lymphedema
}

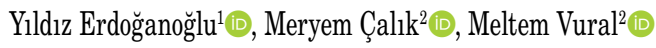 \\ ${ }^{1}$ Department of Physiotherapy and Rehabilitation, Üsküdar University, Faculty of Health Sciences, Istanbul, Turkey \\ ${ }^{2}$ Department of Physical Medicine and Rehabilitation, University of Health Sciences, Bakırköy Dr. Sadi Konuk Training and Research Hospital, Istanbul, Turkey
}

Received: April 18, 2019 Accepted: January 12, 2020 Published online: March 04, 2021

\begin{abstract}
Objectives: In this study, we aimed to investigate the availability of the Timed Up and Go (TUG) test in daily practice instead of the Tampa Scale for Kinesiophobia (TSK) test for the evaluation of fear of movement and to assess the functionality of the upper extremity in postmastectomy lymphedema patients.

Patients and methods: Between March 2018 and July 2018, a total of 30 female patients (mean age $53.8 \pm 12.3$ years; range, 35 to 80 years) with postmastectomy lymphedema were included in this study. The severity of lymphedema of the patients was measured circumferentially at $5-\mathrm{cm}$ intervals. All patients were evaluated for upper extremity functionality using the Timed Functional Arm and Shoulder Test, hand grip strength using a hand dynamometer, and pinch strength using a pinchmeter. The TSK test was used for the evaluation of fear of movement and TUG test was used for the evaluation of functional status of lower extremity.

Results: There was a significant difference in functionality between the affected and unaffected side of upper extremity ( $<<0.05$ ). According to the TSK results, all patients described themselves as kinesiophobic, and advanced fear of movement was found in $66.67 \%$ of the patients. However, according to the TUG scores, lower extremity functionality of all patients was normal.

Conclusion: Our study results showed that, independently of the severity of lymphedema, fear of movement was seen in every patient and functionality of upper extremity decreased on the affected side. On the other hand, the TUG test may not be useful to asses fear of movement in daily practice regarding to functional scores of these patients.
\end{abstract}

Keywords: Lymphedema, kinesiophobia, Timed Up and Go test.

Lymphedema due to mastectomy is likely to result in pain, fatigue, swelling, infection, susceptibility to infection, sensory problems, cosmetics deformity, and significant decrease in functioning of the extremities. ${ }^{[1-5]}$ Previous studies have proved that mastectomy-driven lymphedema is related to shoulder pain and dysfunction of upper extremity and hand. ${ }^{[6-9]}$ Furthermore, decreased arm swing of upper extremity due to lymphedema may influence walking and balance function of lower extremities. ${ }^{[10-13]}$

Kinesiophobia is a condition in which a patient feels insecure during movement and, due to the fear of physical injuries, the patient has unreasonable avoiding of movements. If the patient has any injuries or falls, the situation may become even worse and adversely affect functionality. ${ }^{[14-16]}$ Although the relationship between lymphedema and kinesiophobia has been already demonstrated in the literature, ${ }^{[17]}$ there are few studies which refer to the relationship between functional tests and fear of movement. Functional tests for upper extremity are used for the evaluation of lymphedema patients; however, we are not aware of lower extremity functions in this group. Previously, functional level of lower extremity in different pathologies was evaluated with the Timed Up and Go (TUG) test. ${ }^{[18-21]}$ In the present study, we aimed to investigate the utility of TUG test instead of the Tampa Scale of Kinesiophobia (TSK) for the evaluation of

Corresponding author: Ylldız Erdoğanoğlu, MD. Antalya Bilim Üniversitesi, Sağlık Bilimleri Fakültesi, Fizyoterapi ve Rehabilitasyon Bölümü, 07190 Döşemealtı, Antalya, Türkiye. e-mail: yildiz.erdoganoglu@gmail.com 
kinesiophobia and to assess functionality of the upper extremity in postmastectomy lymphedema patients.

\section{PATIENTS AND METHODS}

This prospective study included a total of 30 female patients (mean age $53.8 \pm 12.3$ years; range, 35 to 80 years) who were admitted to Lymphedema Unit of our center after unilateral mastectomy for breast cancer between March 2018 and July 2018. All patients completed cancer-driven chemotherapy and radiotherapy treatment at least three months ago. Severe pain due to scar of axillary node dissection, malignant lymphedema, relapsing of cancer, infection, orthopedic and neurological disorders resulting in limitation of the upper and lower extremity functions were considered exclusion criteria. The duration of lymphedema varied between one and 240 months. A written informed consent was obtained from each patient. The study protocol was accepted by the Üsküdar University, Faculty of Medicine, Ethics Committee of Non-invasive Clinical Research (B.08.6. YÖK.2.ÜS.0.05.0.06/2018/425 23.02.2018). The study was conducted in accordance with the principles of the Declaration of Helsinki.

All patients were evaluated for age, sex, body mass index, lymphedema duration, and adjuvant treatment. For edema severity, perimetry was performed on both arms at $5-\mathrm{cm}$ intervals from nail root up to shoulder, while the individuals lay on their back, arms rest next to the body and elbows flat. A difference of 1 to $2 \mathrm{~cm}$ was considered mild lymphedema, a difference of 3 to $5 \mathrm{~cm}$ moderate lymphedema, and a difference above $5 \mathrm{~cm}$ severe lymphedema. ${ }^{[22,23]}$

The Upper extremity functionality was assessed using the Timed Functional Arm and Shoulder Test (TFAST). ${ }^{[24]}$ The test is composed of three basic tasks covering a range of motion of joint, strength, and loss of strength. Each task is followed for each arm with regardless of side.

a) Hand to head and back (HHB): Starting position of the test is when the arm rests next to the body. Upon starting of the test, individuals are asked to touch, first, the occipital area with any part of their hand, then to lower their arms and touch any part of the back with dorsum of the hand. Movement is repeated during the test, and every touch on the occipital area is recognized as one set. Individuals are asked to repeat the movement as many as they can in $30 \mathrm{sec}$, and the number of repeats is recorded for both arms. b) Wall wash (inward and outward) motion: A circle of $30.48 \mathrm{~cm}$ in diameter is drawn on a flat wall whereby the shoulder height is taken as the center. The lines are drawn $15.24 \mathrm{~cm}$ above, below, right, and left of the circle for the circle. Individuals are asked to move their arms with circular movements at shoulder-high on the wall. The hand is supported with a towel to reduce friction against the wall. The test starts from the upper line, when the towel is in full contact with the wall. Individuals are asked to pass the arm on all four lines while rotating the wall outwards; every pass of the hand on the upper line is accepted as one set. Individuals are asked to repeat the movement as many as they can in $60 \mathrm{sec}$, and the number of repeats is recorded for both arms. The test is repeated by starting from the upper line and rotating the hand inwards.

c) Gallon jug lift: The test is performed on a mechanism having a counter with $91.44 \mathrm{~cm}$ off the ground, and a shelf above $50.8 \mathrm{~cm}$ to the counter. Individuals are asked to pick up a $3.78-\mathrm{kg}$ gallon from the counter height, slightly touching it on the shelf and lower back to the counter height again. They are asked to repeat the movement as many as they can for $30 \mathrm{sec}$ without leaving the gallon on the shelf. Each touch on the shelf is accepted as one set, and the number of repeats is recorded for $30 \mathrm{sec}$. For each task, total repetitions are recorded and used to calculate the total TFAST scores so that all tasks can be represented equally for a 30 -sec period. For the wall-wash task, the repetitions for the inward and outward directions are added and were, then, divided by 4 to represent $30 \mathrm{sec}$ of data: [HHB + (wall wash inward and outward/4) + gallon jug lift].

Assessment of hand grip and pinch strength was performed at baseline using a hand dynamometer and pinchmeter with high validity and reliability similar to previous studies. ${ }^{[25,26]}$ It was performed on shoulder adduction and neutral rotation in the sitting position with the elbow at $90^{\circ}$ flexion, forearm at mid-rotation, and in a supported position, wrists in neutral position. According to the test procedure, three measurements were taken for the hand grip and pinch strengths, each with one-min intervals between for hand grip and pinch strengths. The median readings were recorded in Newton $(\mathrm{N})$. The measurements were taken for both upper extremities. ${ }^{[25,26]}$

The functional level was measured with the TUG test. First, the test was demonstrated in practice to the patients. The patients were asked to stand up with no support from the chair, to walk at maximal speed 
for $3 \mathrm{~m}$, to turn around another chair again with no support, and to sit back on the first chair. The test was repeated three times, and times of the test were averaged and recorded in sec. ${ }^{[27]}$

Kinesiophobia was assessed using the Turkish version of Tampa Scale for Kinesiophobia (TSK). ${ }^{[28]}$ The scale is composed of 17 questions, and one can get a score, ranging from 17 to 68 . The scores above $\geq 40$ indicate a high level of fear of movement, while scores below $<40$ indicate a low level of fear of movement. ${ }^{[29]}$

\section{Statistical analysis}

Sample size calculation was performed using the $G^{*}$ Power version 3.1.9.2 software (Heinrich-HeineUniversität Düsseldorf, Düsseldorf, Germany). In the structured sample size analysis, the alpha meaning level (Type I error; $\alpha=0.05$ ) was the power value which was required for this study (Type II error; ie $\beta=0.90$ ). A $|\rho|=0.50$ effect size value which was accepted as a high effect width according to the Cohens standards was calculated. ${ }^{[17]}$ The effect width was calculated as $|\rho|=0.50$, and the number of patients needed was calculated as at least 28 . Considering a reasonable dropout rate, we included a total of 30 patients in the study.

Statistical analysis was performed using the IBM SPSS version 23.0 software (IBM Corp., Armonk,
NY, USA). The Shapiro-Wilk test was used to analyze the normality assumption. Descriptive data were expressed in mean \pm standard deviation (SD), median (interquartile range [IQR] $25^{\text {th }}-75^{\text {th }}$ ), or number and frequency. The paired samples t-test was used to compare TFAST scores for affected and unaffected side, hand grip and pinch strength. The Kruskal-Wallis test was used for multiple comparisons according to the severity of lymphedema. The Spearman's correlation analysis was used to identify the relationship between TUG and TSK results. A $p$ value of $<0.05$ was considered statistically significant.

\section{RESULTS}

Demographic and clinical characteristics of the patients are shown in Table 1.

A significant difference was found between the affected side upper extremity functionality and hand grip and pinch strengths compared to the unaffected side upper extremity functioning and hand grip and grip strengths $(\mathrm{p}<0.05)$ (Table 2$)$.

A total of $66.67 \%$ of the patients $(n=20)$ had a high level of fear of movement, while $33.33 \%$ of the patients $(n=10)$ had a low level of fear of movement. According to the severity of lymphedema, nine patients

\begin{tabular}{|c|c|c|c|c|c|}
\hline \multicolumn{6}{|c|}{$\begin{array}{c}\text { TABLE } 1 \\
\text { Demographic and clinical characteristics of patients }\end{array}$} \\
\hline & $\mathrm{n}$ & $\%$ & Mean \pm SD & Median & Min-Max \\
\hline \multicolumn{6}{|l|}{ Edema severity } \\
\hline Mild & 11 & 36.70 & & & \\
\hline Moderate & 11 & 36.70 & & & \\
\hline Severe & 8 & 26.60 & & & \\
\hline Age (year) & & & $53.8 \pm 12.3$ & 53.00 & $35-80$ \\
\hline Height $(\mathrm{cm})$ & & & $158.4 \pm 5.5$ & 158.00 & $150-170$ \\
\hline Body mass index $\left(\mathrm{kg} / \mathrm{m}^{2}\right)$ & & & $28.8 \pm 5.1$ & 28.00 & $20.55-41.32$ \\
\hline Body weight (kg) & & & $72.1 \pm 11.3$ & 72.00 & $54.00-98.00$ \\
\hline
\end{tabular}

\begin{tabular}{|c|c|c|c|}
\hline \multicolumn{4}{|c|}{$\begin{array}{l}\text { TABLE } 2 \\
\text { Functional results of upper extremity after mastectomy }\end{array}$} \\
\hline & Affected side & Unaffected side & \\
\hline & Mean \pm SD & Mean \pm SD & $p$ \\
\hline Hand grip strength & $42.9 \pm 10.8$ & $37.1 \pm 12.4$ & $0.001^{*}$ \\
\hline Pinch strength & $10.0 \pm 3.2$ & $10.7 \pm 3.3$ & $0.024^{*}$ \\
\hline Timed Functional Arm and Shoulder Test & $42.9 \pm 11.0$ & $49.8 \pm 10.1$ & $<0.001^{\star}$ \\
\hline
\end{tabular}




\begin{tabular}{|c|c|c|c|c|}
\hline \multicolumn{5}{|c|}{$\begin{array}{c}\text { TABLE } 3 \\
\text { All patients' fear of movement according to the severity of lymphedema }\end{array}$} \\
\hline & \multicolumn{2}{|c|}{ High level Kinesiophobia } & \multicolumn{2}{|c|}{ Low level Kinesiophobia } \\
\hline & $\mathrm{n}$ & $\%$ & $\mathrm{n}$ & $\%$ \\
\hline \multicolumn{5}{|c|}{ Severity of lymphedema } \\
\hline Mild & 9 & 30.00 & 2 & 6.67 \\
\hline Moderate & 7 & 23.33 & 4 & 13.33 \\
\hline Severe & 4 & 13.33 & 4 & 13.33 \\
\hline Total & 20 & 66.67 & 10 & 33.33 \\
\hline
\end{tabular}

\begin{tabular}{|c|c|c|c|c|c|c|c|c|c|}
\hline & & TSK & d TUG res & TAB & $\begin{array}{l}4 \\
\text { to severit }\end{array}$ & of lymphede & & & \\
\hline & & Mild ( $n=1$ & & & oderate ( $\mathrm{n}$ & & & Severe $(n=$ & \\
\hline & Mean \pm SD & Median & Q1-Q3 & Mean \pm SD & Median & Q1-Q3 & Mean \pm SD & Median & Q1-Q3 \\
\hline TSK & $44.2 \pm 7.9$ & 43.00 & $42.00-46.00$ & $40.5 \pm 8.6$ & 40.00 & $38.00-45.00$ & $42.0 \pm 7.6$ & 40.50 & $35.75-48.50$ \\
\hline TUG & $9.0 \pm 1.2$ & 8.86 & $8.33-10.07$ & $8.3 \pm 1.3$ & 8.14 & $7.02-9.33$ & $9.8 \pm 2.6$ & 9.29 & $7.47-12.01$ \\
\hline
\end{tabular}

(30.00\%) with mild lymphedema had a high-level fear of movement, while two patients $(6.67 \%)$ had a low-level fear of movement. Seven patients (23.33\%) with moderate lymphedema had a high-level fear of movement, while four patients (13.33\%) had a low-level fear of movement. Four patients (13.33\%) with severe lymphedema had a high-level fear of movement, while four patients (13.33\%) had a low-level fear of movement (Table 3).

On the other hand, the TSK and TUG scores did not significantly differ according to the severity of lymphedema ( $>0.05)$ (Table 4). While the TSK scores suggested the fear of movement in all lymphedema groups, the TUG scores were within normal limits in all lymphedema groups. In addition, there was no significant correlation between the TSK and TUG scores in patients with postmastectomy lymphedema ( $p>0.05)$.

\section{DISCUSSION}

To date, few studies have examined the relationship between lymphedema due to mastectomy and fear of movement. ${ }^{[17,30]}$ However, there are no specific tests for the evaluation of kinesiophobia in this patient group. To the best of our knowledge, there is no study in which TSK with a functional test was used in clinical aspect, while evaluating the fear of movement in postmastectomy lymphedema patients. Therefore, in our study, we attempted to evaluate the practical results of TSK with the TUG functional test. Our study results showed that the upper extremity functionality significantly decreased on the affected side and majority of the patients had a high level of fear of movement according to TSK. In addition, the severity of lymphedema did not make a significant difference on the TUG scores and there was no significant difference between the patients who had a low or high level of fear of movement.

In previous studies evaluating upper extremity functionality, the surveys such as Disabilities of the Arm, Shoulder and Hand, Shoulder Pain and Disability Index, and shoulder range of motion and muscle strength, elbow muscle strength, wrist grip, and compression force are used. ${ }^{[7-11]}$ Some tests to measure functional performance have been described in the literature. Most of these tests only include elevationbased tasks rather than internal/external rotation, which is critical for shoulder function. However, there is no specific functionality test for patients who have lymphedema after mastectomy. In our study, the TFAST, which involved movements and activities that patients frequently performed in their daily lives, was used for evaluating upper extremity functionality. Also, the wrist and pinch strengths were evaluated. The TFAST has been shown to provide a realistic estimate of a person's functional performance during clinical evaluation and may allow for longitudinal tracking during treatment in which operated side and unaffected side shoulder functions of patients after the breast cancer surgery can be compared. It is also demonstrated that patients have a loss of functionality on the operated side. ${ }^{[8,9,23,31,32]}$ In their study, Smooth et al. ${ }^{[8]}$ examined upper extremity dysfunctions after breast cancer surgery and reported that it 
was more common for women with lymphedema to have shoulder muscle strength loss, joint range of motion loss, elbow, wrist and hand grip strength loss, compared to women without lymphedema. The authors further showed that the most significant difference was in the shoulder abduction range of motion and grip strength. Likewise, a study by Park et al. ${ }^{[9]}$ demonstrated that upper extremity functions reduced in patients with lymphedema after breast cancer operation. The authors indicated that the area where the surgical intervention and radiotherapy were applied after breast cancer was a critical area for functional use of the arm, and that it could affect the muscle groups involving the scapulohumeral rhythm, which is needed for functional shoulder movement. A study by Shamley et al. ${ }^{[31]}$ also examined the final changes in the shoulder muscle movement after breast cancer treatment and found a significant relationship between the activity in upper trapezius muscles and rhomboid muscle and functionality. In addition, disability increased with the increased pain in the shoulder. In our study, the results of difference of functionality between the affected side and unaffected side are consistent with previous studies in the literature.

Kinesiophobic patients often avoid movements and activities and, if the patient has any injuries or falls, this makes the situation even worse and adversely affects functionality. There are many reports in the literature about the fear of movements which are mainly associated with pain. ${ }^{[14-16]}$ To the best of our knowledge, there is a limited number of studies evaluating the effects of kinesiophobia in patients with lymphedema after mastectomy. ${ }^{[17,30]}$ The study by Karadibak et al. ${ }^{[30]}$ found a strong relationship between severity of lymphedema and kinesiophobia. Gencay Can et al. ${ }^{[17]}$ also observed a significant relationship between kinesiophobia and the presence of lymphedema; however, they found no significant correlation between kinesiophobia and the stage and severity of lymphedema in their study. The authors also showed that $76 \%$ of patients with lymphedema after mastectomy had a high-level fear of movement. However, in the literature, there are no other data available to compare this rate. We believe that differences between high fear of movement rates from different studies are related to cut-off values used. However, generally accepted cut-off value in this subject is 40 , and higher values may be considered as high fear of movement. ${ }^{[29]}$ Therefore, in our study, we accepted this value as the cut-off value.
The TUG test is used to evaluate functional level of lower extremity in different pathologies and needs a clinician monitoring. ${ }^{[33,34]}$ Different normal values, depending on the age and pathology, have been used for TUG test. ${ }^{[35-37]}$ However, the cut-off value for this test is usually accepted as $12 \mathrm{sec}^{[20]}$

Kinesiophobia may be one of the important causes which affects functioning in the patients with lymphedema after mastectomy. In our study, we studied functional test's validity in daily clinical practice as an additional indicator for the evaluation of functionality in patients with postmastectomy lymphedema. Using the cut-off values in our study, we found that the patients had no significant functional loss in their lower extremities due to fear of movement.

The main limitations of the present study include ignoring duration of lymphedema due to the chosen pathological group features, regarding limitation of number, but not separately researching the effects of additional treatments applied to lymphedema patients, and not supporting the functional level with another test. Further studies which would be conducted considering these limitations may offer more accurate results about functional conditions of these patients.

In conclusion, our study results showed that the upper extremity functionality of the affected side decreased in lymphedema patients after mastectomy and there was fear of movement in all patients. In this patient group, therefore, regardless of the severity of lymphedema and the degree of kinesiophobia, the TUG test may not be useful in assessing fear of movement in daily practice.

\section{Declaration of conflicting interests}

The authors declared no conflicts of interest with respect to the authorship and/or publication of this article.

Funding

The authors received no financial support for the research and/or authorship of this article.

\section{REFERENCES}

1. de Jong N, Candel MJ, Schouten HC, Abu-Saad HH, Courtens AM. Prevalence and course of fatigue in breast cancer patients receiving adjuvant chemotherapy. Ann Oncol 2004;15:896-905.

2. Calitchi E, Kirova YM, Otmezguine Y, Feuilhade F, Piedbois Y, Le Bourgeois JP. Long-term results of neoadjuvant radiation therapy for breast cancer. Int $\mathrm{J}$ Cancer 2001;96:253-9.

3. Rockson SG. Lymphedema. Am J Med 2001;110:288-95.

4. Cohen SR, Payne DK, Tunkel RS. Lymphedema: strategies for management. Cancer 2001;92:980-7. 
5. Sagen A, Kaaresen R, Sandvik L, Thune I, Risberg MA. Upper limb physical function and adverse effects after breast cancer surgery: a prospective 2.5-year follow-up study and preoperative measures. Arch Phys Med Rehabil 2014;95:875-81.

6. Katz J, Poleshuck EL, Andrus CH, Hogan LA, Jung BF, Kulick DI, et al. Risk factors for acute pain and its persistence following breast cancer surgery. Pain 2005;119:16-25.

7. Bosompra K, Ashikaga T, O'Brien PJ, Nelson L, Skelly J. Swelling, numbness, pain, and their relationship to arm function among breast cancer survivors: a disablement process model perspective. Breast J 2002;8:338-48.

8. Smoot B, Wong J, Cooper B, Wanek L, Topp K, Byl N, et al. Upper extremity impairments in women with or without lymphedema following breast cancer treatment. J Cancer Surviv 2010;4:167-78.

9. Park JE, Jang HJ, Seo KS. Quality of life, upper extremity function and the effect of lymphedema treatment in breast cancer related lymphedema patients. Ann Rehabil Med 2012;36:240-7.

10. Yang JL, Lin JJ. Reliability of function-related tests in patients with shoulder pathologies. J Orthop Sports Phys Ther 2006;36:572-6.

11. Kumta P, MacDermid JC, Mehta SP, Stratford PW. The FITHaNSA demonstrates reliability and convergent validity of functional performance in patients with shoulder disorders. J Orthop Sports Phys Ther 2012;42:455-64.

12. Bertels T, Schmalz T, Ludwigs E. Biomechanical influences of shoulder disarticulation prosthesis during standing and level walking. Prosthet Orthot Int 2012;36:165-72.

13. Ford MP, Wagenaar RC, Newell KM. Arm constraint and walking in healthy adults. Gait Posture 2007;26:135-41.

14. Vlaeyen JW, Linton SJ. Fear-avoidance and its consequences in chronic musculoskeletal pain: a state of the art. Pain 2000;85:317-32.

15. Nederhand MJ, Ijzerman MJ, Hermens HJ, Turk DC, Zilvold G. Predictive value of fear avoidance in developing chronic neck pain disability: consequences for clinical decision making. Arch Phys Med Rehabil 2004;85:496-501.

16. Swinkels-Meewisse EJ, Swinkels RA, Verbeek AL, Vlaeyen JW, Oostendorp RA. Psychometric properties of the Tampa Scale for kinesiophobia and the fear-avoidance beliefs questionnaire in acute low back pain. Man Ther 2003;8:29-36.

17. Gencay Can A, Can SS, Ekşioğlu E, ÇakcıFA. Iskinesiophobia associated with lymphedema, upper extremity function, and psychological morbidity in breast cancer survivors? Turk J Phys Med Rehabil 2018;65:139-46.

18. Sebastião E, Sandroff BM, Learmonth YC, Motl RW. Validity of the Timed Up and Go Test as a Measure of Functional Mobility in Persons With Multiple Sclerosis. Arch Phys Med Rehabil 2016;97:1072-7.

19. van Hedel HJ, Wirz M, Dietz V. Assessing walking ability in subjects with spinal cord injury: validity and reliability of 3 walking tests. Arch Phys Med Rehabil 2005;86:190-6.

20. Singh DK, Pillai SG, Tan ST, Tai CC, Shahar S. Association between physiological falls risk and physical performance tests among community-dwelling older adults. Clin Interv Aging 2015;10:1319-26.

21. Lusardi MM, Fritz S, Middleton A, Allison L, Wingood M, Phillips E, et al. Determining Risk of Falls in Community
Dwelling Older Adults: A Systematic Review and Meta-analysis Using Posttest Probability. J Geriatr Phys Ther 2017;40:1-36.

22. McKenzie DC, Kalda AL. Effect of upper extremity exercise on secondary lymphedema in breast cancer patients: a pilot study. J Clin Oncol 2003;21:463-6.

23. Taylor R, Jayasinghe UW, Koelmeyer L, Ung O, Boyages J. Reliability and validity of arm volume measurements for assessment of lymphedema. Phys Ther 2006;86:205-14.

24. Shah KM, Baker T, Dingle A, Hansmeier T, Jimenez M, Lopez S, et al. Early Development and Reliability of the Timed Functional Arm and Shoulder Test. J Orthop Sports Phys Ther 2017;47:420-31.

25. Haidar SG, Kumar D, Bassi RS, Deshmukh SC. Average versus maximum grip strength: which is more consistent? J Hand Surg Br 2004;29:82-4.

26. Halpern CA, Fernandez JE. The effect of wrist and arm postures on peak pinch strength. J Hum Ergol (Tokyo) 1996;25:115-30.

27. Wall JC, Bell C, Campbell S, Davis J. The Timed Get-upand-Go test revisited: measurement of the component tasks. J Rehabil Res Dev 2000;37:109-13.

28. Tunca Yılmaz Ö, Yakut Y, Uygur F, Uluğ N. Tampa Kinezyofobi Ölçeği'nin Türkçe versiyonu ve test-tekrar test güvenirliği. Fizyoter Rehabil 2011;22:44-9.

29. Domenech J, Sanchis-Alfonso V, López L, Espejo B. Influence of kinesiophobia and catastrophizing on pain and disability in anterior knee pain patients. Knee Surg Sports Traumatol Arthrosc 2013;21:1562-8.

30. Karadibak D, Yavuzsen T, Saydam S. Prospective trial of intensive decongestive physiotherapy for upper extremity lymphedema. J Surg Oncol 2008;97:572-7.

31. Shamley DR, Srinanaganathan R, Weatherall R, Oskrochi $\mathrm{R}$, Watson $\mathrm{M}$, Ostlere S, et al. Changes in shoulder muscle size and activity following treatment for breast cancer. Breast Cancer Res Treat 2007;106:19-27.

32. Kaya T, Karatepe AG, Günaydn R, Yetiş H, Uslu A. Disability and health-related quality of life after breast cancer surgery: relation to impairments. South Med J 2010;103:37-41.

33. Merchant CR, Chapman T, Kilbreath SL, Refshauge KM, Krupa K. Decreased muscle strength following management of breast cancer. Disabil Rehabil 2008;30:1098-105.

34. Stienen MN, Ho AL, Staartjes VE, Maldaner N, Veeravagu A, Desai A, et al. Objective measures of functional impairment for degenerative diseases of the lumbar spine: a systematic review of the literature. Spine J 2019;19:1276-93.

35. Nocera JR, Stegemöller EL, Malaty IA, Okun MS, Marsiske M, Hass CJ. Using the Timed Up \& Go test in a clinical setting to predict falling in Parkinson's disease. Arch Phys Med Rehabil 2013;94:1300-5.

36. Steffen TM, Hacker TA, Mollinger L. Age- and genderrelated test performance in community-dwelling elderly people: Six-Minute Walk Test, Berg Balance Scale, Timed Up \& Go Test, and gait speeds. Phys Ther 2002;82:128-37.

37. Struble-Fitzsimmons D, Pinto Zipp G, DeLuca D, Zhang N. Exploring the Relationship Between Timed Up and Go Test Times and Falls in an Inpatient Geriatric Psychiatry Unit: A Retrospective Case-Control Study. J Geriatr Phys Ther 2019. [Epub ahead of print] 